\title{
Zur Problematik der Datierung von Würm-Sedimenten auf der Basis paläontologischer Funde
}

\author{
Von Rudolf Musil, Brno/Brünn
}

Zusammenfassung: Der Artikel handelt über die Methodik der pleistozänen Forschungen im Terrain und über die Art der Bearbeitung paläontologischen Materials. Er zeigt die Möglichkeiten der Benutzung dieser Funde für die Stratigraphie der Würm-Sedimente. Auf Grund einer eingehenden morphologischen und metrischen Analyse der ganzen Faunen-Gemeinschaft ist es möglich die betreffende stratigraphische Einreihung durchzuführen. Die Faunen-Gemeinschaften des W 1/2-Interstadials und des R/W-Interglazials sind einander sehr ähnlich. Nach dem W 1/2Interstadial tritt eine große faunistische Veränderung ein, und bis zum Ende des Würms ändert sich dann diese Gesellschaft nicht mehr. Das Ende des ersten Würm-Interstadials ist also eine sehr wichtige Grenze für die Änderung der ganzen Faunen-Gemeinschaft. Am Ende des Artikels werden dann einige stratigraphische Fragen erörtert.

Summary: The article deals with the methodology of the Pleistocene palaeontological research in the field as well as with the way in which the collected material is being treated. It shows the possibilities of using the paleontological material for the stratigraphy of the Würm sediments. By virtue of a thorough morphological and metrical analysis of the entire faunal community the respective stratigraphic classification can be carried out. The faunal community of the interstadial period $\mathrm{W} 1 / 2$ and of the interglacial $\mathrm{R} / \mathrm{W}$ are very similar to each other. After the sedimentation of the fossil soil of W $1 / 2$ a great faunal change took place and until the end of the last glacial period the faunal community which arose almost did no more change. Therefore, the end of this period is an important boundary of the fauna. Finally the article deals with some stratigraphic questions.

Das letzte Glazial ist ein relativ sehr kurzer Zeitabschnitt, weist aber dabei eine von großen Klima-Ausschlägen beeinflußte ausdrucksvolle Gliederung auf. Bisher ließ man die Festsetzung des Zeitpunktes der einzelnen Interstadiale und Stadiale wegen der Funde größerer Säugetiere entweder gar nicht zu oder aber betrachtete diese als unsicher. Soweit diese Festlegung doch vorgenommen wurde, sind dabei nur die Arten der Leitfossilien, hauptsächlich Dicerorbinus kirchbergensis und Palaeoloxodon antiquus berücksichtigt worden, von denen man annahm, daß sie im Letzten Interglazial ausstarben. Auch dann wurde manchmal auf die Veränderungen in der Gesamtgemeinschaft, die zufolge klimatischer Oszillationen gewiß eintreten, hingewiesen; zumeist wurde aber diese Tatsache nur vermerkt. Ich vermute, daß dies daher kommt, weil von vielen übertrieben betont wurde, daß die erhaltengebliebenen Funde nur einen Teil aller damals lebenden Tiere darstellen. Die zweite und hauptsächlichste Behinderung aber ergab sich daraus, daß es nur sehr wenige Lakolitäten gibt, in denen die Funde präzis nach einzelnen Horizonten gesammelt worden sind. Bei älteren Funden wurde diese detaillierte Horizontierung nicht durchgeführt; bei den neueren Forschungen kam es bisher entweder noch nicht zu deren Verarbeitung oder aber wurde diese mit den alten Arbeitsmethoden vorgenommen.

Versuchen wir jetzt das ganze Problem, das mit der Gliederung der Würm-Sedimente auf Grund der Säugetierfunde zusammenhängt, aufzuspalten, bis zu welchem Ausmaß es möglich ist, zu diesem Ziele zu gelangen, und unter welchen Bedingungen und Voraussetzungen. Das ganze Problem können wir in mehrere Teile zerlegen. Zuerst sind es einmal die Funde der Leitfossilien-Arten wie z. B. D. kirchbergensis, P. antiquus, Hystrix u. ä., die in unseren Gebieten im Letzten Interglazial ausstarben und uns so die oberste Grenze ihrer Verbreitung anzeigen. Diese Methode ist die einfachste, wird auch hauptsächlich angewandt, vorausgesetzt, daß die angeführten Arten in den Sedimenten vorhanden sind.

Eine weitere Möglichkeit zur stratigraphischen Einordnung der Schichten beruht auf der Auswertung der Gesamtgemeinschaft. Voraussetzung für dieses ist allerdings eine 
detaillierte Aufklärung des paläontologischen Materials aus den Sedimenten und dessen Vorkommen in einer größeren Menge. Diese beiden Voraussetzungen sind hauptsächlich bei Funden aus Kulturschichten und bei solchen aus Höhlensedimenten gegeben. Vereinzelte Funde oder Knochenfunde in kleinerer Anzahl wie z. B. in Lössen, die verhältnismäßig häufig sind, kommen nicht in Betracht. Die Anwendung dieser Methode, d. i. die Auswertung der Gesamtgemeinschaft, gliedert man in zwei Teile. Einerseits ist es die Bestimmung aller aufgefundenen Knochen und auf dieser Grundlage dann die prozentuelle Berechnung der vorhandenen Arten. Auf diese Weise erhalten wir ein Bild bestimmter Veränderungen, das in einigen Fällen aber gewiß von der Auswahl der Faktoren, welcher Art auch immer, beeinflußt ist. Trotz dieser sich negativ äußernden Tendenzen bedeutet diese Analyse der Fauna einen bestimmten Fortschritt, der eine erweiterte Anwendung zur gröberen stratigraphischen Einreihung der Schichten ermöglicht. Auch sehen wir beim Studium mehrerer Lokalitäten unter Anwendung dieser Methode, daß die durch die Auswahl verursachten Eingriffe nicht so wesentlich sind, als man vermuten könnte. Es ist dies heute die zumeist angewandte Methode der Bearbeitung des paläontologischen Materials. Sie basiert auf der Voraussetzung der klimatischen Veränderungen, welche so groß sind, daß sie Veränderungen auch in der Zusammensetzung des Pflanzenreiches und damit auch im Tierreich hervorrufen. Diese Methode führte zur Überwindung der überlebten Anschauung, daß sich die Tiere während des ganzen Letzten Glazials nicht veränderten und daß deshalb auch jede Arbeit, aufgewandt zur deren genauer Bestimmung, für die Stratigraphie überflüssig, ja nutzlos sei. Es ist selbstverständlich, daß diese Ansichten seiner Zeit zu einer ausweglosen Resignation führten. Und interessant ist es, daß sie auch heute noch bei vielen mit der Erforschung des Quartärs sich befassenden Spezialisten verhältnismäßig tief verwurzelt sind.

Der zweite Teil dieser Methode, der notwendigerweise gleichzeitig durchgeführt werden muß, ist die Detail-Analyse der aufgefundenen Fauna sowohl hinsichtlich der metrischen als auch der morphologischen Seite. Auch wenn es sich manchmal stets um die gleichen Arten handelt, treten während des Letzten Glazials Veränderungen in den vertretenen primitiven und progressiven Kennzeichen ein, ob diese nun durch die Entwicklung der betreffenden Art oder durch äußere Bedingungen und deren Veränderungen hervorgerufen wurden. Es ist selbstverständlich, daß diese morphologischen und fallweise auch metrischen Veränderungen nicht von gleicher Intensität bei allen Arten sind. Einige Arten sind weniger plastisch und anpassungsfähig, ihre Kennzeichen bleiben längere Zeit konstant und ändern sich nicht: Andere Arten wiederum sind im Gegenteil anpassungsfähiger; einige ihrer Kennzeichen unterliegen sehr stark Veränderungen und reagieren gut auf Veränderung der Umwelt-Bedingungen. Zusammenfassend ist es möglich zu sagen, daß die Mehrzahl der Arten auf Klimawirkungen während eines längeren Zeitabschnittes sehr empfindlich reagieren; auch können wir z. B. Abweichungen aus der Zeit der Sedimentation der letzten pleistozänen Schwarzerde und zu Ende des Glazials feststellen. Die Summe dieser Abweichungen von einer größeren Anzahl der aufgefundenen Arten, selbstverständlich auf Grund einer größeren Materialmenge, charakterisiert uns dann stets den bestimmten gleichen Zeitabschnitt. Diese Methode setzt jedoch eine weitreichend detaillierte Verarbeitung allen Materials voraus; sie ist auch sowohl zeitlich als auch hinsichtlich der Materialkenntnisse recht anspruchsvoll. Es handelt sich dabei um keine neue Methode. Sie wurde bereits von mehreren Fachleuten angewandt, so z. B. von W. Soergel beim Studium der Hyänen aus der L in d e n t a 1 - Höhle bei Gera im Jahre 1937, von Rode u. a. Neu daran ist nur, daß es sich als notwendig erwies, diese Methode von der Erforschung einzelner Arten auf die Erforschung der Gesamtgemeinschaft, d. h. aller aufgefundenen Tiere, zu übertragen und stratigraphische Schlußfolgerungen nur auf Grund aller gewonnenen Erkenntnisse zu ziehen. Ich nehme an, daß dies heute der richtige Weg ist, der eine präzise stratigraphische Einstufung der Würm-Sedimente erlaubt.

Auf die angeführte Weise habe ich die Fauna des Švéd ův stůl (Schwedentisch- 
grotte), einer Höhle im südlichen Teil des Mährischen Karstes, bearbeitet. Die Höhle Švédův stůl ist ein typischer Höhlenhorst von Hyänen, die anscheinend längere Zeit hier lebten. Ihrem Vorkommen danken wir die große Anzahl der Arten, die beträchtlich größer ist als in Höhlen, die von Höhlenbären besiedelt waren. Ein bestimmter Teil der osteologischen Funde ist notwendigerweise auch der Tätigkeit des Menschen zuzuschreiben, der sich während einiger Zeit in der angeführten Lokalität aufhielt.

Die in der Höhle švéd ův stůl vertretenen Sedimente sind in den einzelnen Schichten sehr ausdrucksvoll gegliedert. Sehr gut läßt sich die Schicht des Letzten WürmLösses (W 3) unterscheiden, auch die bräunlichen Lößerden in ihrem Liegenden (W 2/3) und weiter der Löß W 2, welcher in dem Hangenden der Rendzina-Böden liegt (R/W, W 1/2). Die Rendzina-Böden setzten sich einesteils aus einigen Horizonten schwach gefärbter Kalksteintrümmer zusammen, andernteils aus einzelnen schwachen Schichten, die sich durch verschiedene Nuancen brauner Farbe unterscheiden, und bilden die Oberschicht der gesamten Schichtengruppe.

Während die stratigraphische Einstufung der Löß-Schichten im wesentlichen klar war, rollte der Schichtenkomplex der Rendzina bestimmte Probleme auf. Durch die Forschungen, welche in der letzten Zeit in diesen Lokalitäten durchgeführt worden sind, ergab sich nämlich, daß sich die Arten der Fauna des ersten Würm-Interstadials und die des Letzten Interglazials annähernd gleichen. Es fanden sich jedoch einige Knochen, welche eine Einstufung dieser Böden in das Letzte Interglazial gestatten. Ich aber nehme an, daß es sich nicht um die ganze Masse des angeführten Schichtenkomplexes, sondern um einen bestimmten Teil desselben handelt.

Vorher habe ich erwähnt, daß die einzelnen Schichten der Sedimente sehr gut unterscheidbar sind. Dieser Umstand - gut erhaltene und unterscheidbare einzelne Schichten ist für das Studium der paläontologischen Funde sehr bedeutsam, ebenso für die Möglichkeit der präzisen Trennung, welche uns die Veränderungen in der Faunen-Gemeinschaft während der einzelnen Zeitabschnitte und die Abhängigkeit der einzelnen Arten von den Klimaten u. ä. aufzuzeigen gestattet. Durch neuere Forschungen wurde die Fauna präzise horizontiert; die Anzahl der Arten ist relativ groß, ebenso auch die Anzahl der Einzelwesen. Es steht demnach dem Aufzeigen der Entwicklung der Fauna in diesem Gebiet nichts im Wege.

Richten wir unser Augenmerk vorerst auf die Faunen-Gemeinschaft in der Schicht der dunklen, humusartigen Erden. In braunen Lehmen wurden folgende Arten vorgefunden: Lepus timidus, Castor fiber, Panthera spelaea, Felis silvestris, Crocuta spelaea, Canis lupus, Vulpes vulpes, Alopex lagopus oder Vulpes corsac, Gulo gulo, Lutra lutra, Meles meles, Mustela martes, Ursus spelaeus, Mammonteus primigenius, Tichorbinus antiquitatis, ?Dicerorbinus sp., Sus scrofa, Cervus elaphus, Alces alces, Megaceros sp., Rangifer sp., Bos primigenius, Rupicapra rupicapra, Equus mosbachensis-abeli-Gruppe, Equus germanicus, Equus (A.) hydruntinus, Equus cf. gmelini, Ovis sp. oder Capra sp., Marmota sp., durch Hystrix benagelte Knochen. Die größte Anzahl weisen Höhlenbär, Pferd und Wollhaariges Nashorn auf. Die übrigen Arten sind schon weniger vertreten.

Verweilen wir bei einigen Arten, bei den Ergebnissen ihrer genauen metrischen und morphologischen Analyse. Aus gewissen Gründen wäre eine allzu große Ausweitung dieses Kapitels, beziehungsweise eine Erörterung aller aufgefundenen Arten, nicht angebracht.

Eines der bedeutsamsten studierten Objekte der erhalten gebliebenen Reste von Höhlenhyänen sind deren Zähne. Besonders aufschlußreich ist an den unteren Reißzähnen der Teil hinter dem Protoconid, der verschieden entwickelt zu sein pflegt und uns das Entwicklungsstadium dieser Tiere gut aufzeigt. Ebenso lassen sich am letzten unteren Prämolar primitive und fortgeschrittene Anzeichen erkennen und zwar in der Morphologie der 
Krone und auch in deren Ausmaßen. Die Zähne der Höhlenhyänen können uns demnach zur groben Unterscheidung des Alters der zugehörigen Sedimente sehr gut dienen.

Verhältnismäßig häufig finden sich in den Schichten des Š véd ů v s t ů 1 die Höhlenbären. Die Zähne derselben sind zumeist erhalten geblieben, bei denen ich außer der morphologischen Analyse auch die variations-statistische Methode anwandte. Die morphologische Analyse führte ich hauptsächlich nach K. EHREnberg durch, bei der metrischen Statistik hielt ich mich an die Arbeiten K. Rode's und W. Soergel's.

Die Zähne der Höhlenbären eignen sich ihrer großen Variabilität halber nicht gut zur Lösung der oben angeführten Aufgabe; dennoch wurden sie aber verwendet. Im allgemeinen war es auch in dieser Lokalität möglich, die große Variabilität bei den einzelnen Zähnen festzustellen; es ergaben sich aber auch bestimmte Abweichungen zwischen den einzelnen Schichten. So z. B. waren beim zweiten oberen Molar die Zähne aus dem W 2 gewissermaßen mehr arktoid gelagert als die Zähne aus dem braunen Lehm. Ihre Ausmaße waren im Durchschnitt auch etwas kleiner. Ein markanter Unterschied existierte in der Metaconlänge. Die gleichen verkleinerten Ausmaße in der Schicht W 2 konnten wir auch bei dem ersten oberen Molar beobachten. Ansonsten war die morphologische Variabilität dieses Zahnes um etwas geringer als bei $\mathrm{M}^{2}$. Ahnlich wie bei dem letzten oberen Zahn ist die Variabilität auch bei dem letzten unteren Zahn stark. Recht häufig kommen in dem Schichtkomplex braunen Lehms auch Molare mit einer geringen Differenzierung des mittleren Feldes vor, was sich mehr als ein bestimmtes arktoides Zeichen bewerten läßt. Was die anderen unteren Zähne anlangt, unterscheiden sie sich in beiden Hauptschichten, d. i. in Schichten mit der größten Fundmenge (Schichtkomplex brauner Erden und Löß-W 2), die durch Entoconid gebildet wurden. Man kann wiederum beobachten, daß die Längsmaße der Zähne aus dem W 2 geringer sind als aus den braunen Lehmen. Ahnliche geringere oder größere Abweichungen können wir auch bei weiteren Zähnen feststellen.

Es gelang uns also bei Funden aus dem Rendzina-Schichtkomplex und aus dem Löß W 2 an den Zähnen der Höhlenbären bestimmte Abweichungen nachzuweisen. Diese Deduktionen kann man allerdings nicht verallgemeinern, sie gelten nur für den Š véd ů v $s \mathrm{t}$ ůl, und zu weiteren Folgerungen wird man erst dann gelangen, wenn man auf diese Weise weitere Lokalitäten durchforscht haben wird.

Eines der am häufigsten vertretenen Tiere, das sich für die detaillierte Stratigraphie des Würms mit am besten eignet, ist das P f e $\mathrm{r}$ d. Der morphologische Bau seiner Zähne ändert sich ständig, und bei einer größeren Materialmenge wird es sehr gut möglich sein, präzise das Stadial oder Interstadial, aus welchem das Material herrührt, festzustellen. Die einzelnen Gruppen der Pferde sind nicht in allen Schichten gleich. In der Schichtgruppe der dunklen Böden treten noch Zähne mit relativ primitiven Kennzeichen auf. Es ist möglich, sie in zwei Gruppen einzuteilen. In der einen Gruppe ähneln sie sehr der Pferdegruppe Equus mosbachensis-abeli, und es ist nicht ausgeschlossen, daß sie mit dieser Gruppe identisch sind; in der anderen ist es die Art Equus germanicus.

Die Zähne im W 2 unterscheiden sich vollständig von den Zähnen der vorhergehenden. Sie sind entwicklungsmäßig viel fortgeschrittener, die primitiven Kennzeichen treten stark in den Hintergrund. Bei den unteren Zähnen ist auch eine metrische Abweichung zu sehen; die Zähne aus dem W 2 sind kleiner. Die Gruppe des Equus mosbachensis-abeli kommt in diesem Stadial schon nicht mehr vor. Das Ende der Sedimentation der dunklen Böden zeichnet sich also als eine bedeutsame Grenzscheide ab, welche die Pferdezähne primitiveren Baues von denen der entwicklungsmäßig fortgeschritteneren unterscheidet. Morphologisch unterscheiden sich die Zähne aus dem W 3 stark von jenen, die in den dunklen. Böden vorgefunden wurden, und deren stärkere Wellung des Zahnschmelzes, die größere Länge der Sporne u. ä. lassen die Möglichkeit zu, sie mit dem feuchten Klima zu erklären, welches in dieser Zeit vorherrschte. 
Verhältnismäßig wenig Funde haben wir aus der Schicht des W 2/3. Es scheint aber, das diese den Funden aus der Schichte des W 2 sehr ähnlich sind.

Im dritten Würm-Stadial ist eine Verringerung der Ausmaße offenkundig, wobei diese Verringerung an den Molaren besser erkennbar ist als an den Prämolaren. Stark tritt diese Änderung wiederum in der morphologischen Ausbildung der Kronen hervor. Man kann sagen, daß die betreffenden morphologischen Abweichungen zwischen den Funden im W 2 und im W 3 um vieles stärker und augenfälliger sind als bei den Zähnen aus dem Schichtkomplex der dunklen Böden und aus dem Löß W 2. Auch wenn also die Veränderung in der Morphologie der Zähne zwischen dem Schichtkomplex der Rendzina und dem Löß W 2 mehr grundsätzlicher Natur ist, weil es das Ende des Vorkommens einer größeren Anzahl primitiver Kennzeichen bedeutet, ist die Veränderung im W 2 und im W 3 plötzlicher und das Ausscheiden der Kennzeichen, das im W 2 in Erscheinung tritt, augenscheinlicher und rascher. Die einzelnen Zeitabschnitte sind also durch Pferdegruppen charakterisiert, welche durch die Entwicklung ihrer morphologischen und manchmal auch der metrischen Kennzeichen gut unterscheidbar sind.

Ich halte es nicht für angebracht, mich näher mit dieser Frage, beziehungsweise mit der Erörterung noch weiterer Arten zu befassen. Tatsache ist, daß diese auf den ersten Blick geringfügigen $\mathrm{Abweichungen} \mathrm{keine} \mathrm{besonderen} \mathrm{Variationen} \mathrm{der} \mathrm{Individuen} \mathrm{sind}$ und in ihrer Gesamtheit eine bedeutsame Charakterisierung der Tiere dieses oder jenes Zeitabschnittes bilden. Werden auf diese Weise alle Arten bearbeitet, dann gelangen wir zu guten Voraussetzungen für eine detaillierte chronologische Einstufung der einzelnen Schichten.

Der letzte Zeitabschnitt des Pleistozäns, in dem die Fauna in der schon früher angeführten Zusammensetzung auftritt, ist die Zeit der Bildung der letzten pleistozänen Schwarzerde. Bis zum Ende des Würms existiert dann eine Faunen-Gemeinschaft, die sich von dieser diametral unterscheidet und sich dann bis ans Ende des Würms - abgesehen von dem Aussterben einiger Arten - in ihrer Zusammensetzung nicht wesentlich mehr ändert. Dies bedeutet, daß die klimatische Veränderung, von der das zweite WürmStadial begleitet war, so weitreichend und dabei so langfristig war, daß sie die Vernichtung gewesener Gemeinschaften zustande brachte und von dieser nur jene Tiere ausgenommen waren, welche sich den veränderten Bedingungen anzupassen vermochten oder von einigen neuen klimatischen Faktoren weniger abhängig waren. Aus dem zweiten Würm-Stadial kennen wir aus dem Švéd ův st ůl diese Arten: Lepus sp., Crocuta spelaea, Canis lupus, Vulpes vulpes, Ursus spelaeus, Tichorhinus antiquitatis, Rangifer tarandus, Equus germanicus, Equus cf. gmelini.

Diese Faunen-Gemeinschaft ist wesentlich kärger, alle typischen Wasser- und einige wärmeliebende Tiere verschwinden. Es ist aber nötig hinzuzufügen, daß es sich nicht nur um eine Temperaturänderung handeln konnte, sondern daß die klimatischen Veränderungen weitreichender gewesen sein mïssen. Durch die bloße Temperaturänderung konnte es beispielsweise nicht zum Ausscheiden der Hirsche kommen, die heute etwas nördlicher als $60^{\circ}$ nördlicher Breite leben, also in verhältnismäßig kalten Gebieten. Wir treffen sie auch in der typischen Taiga an; in den Bergen steigen sie in Höhen bis zu $3000 \mathrm{~m}$. Schon heute können wir sagen, daß die Zeit der Bildung der letzten Schwarzerde in unseren Gegenden der letzte Zeitabschnitt des Pleistozäns ist, in dem Auerochs und Bison in Rudeln auftraten, weiter aber auch große Maral-ähnliche Hirsche und Elche, da sie in dieser Zeit anscheinend ihre optimalen Lebensbedingungen hatten. Gegen das Ende des Würms kommen sie nur mehr vereinzelt vor, und eine Gruppe großer Maral-ähnlicher Hirsche tritt schon nicht mehr auf. Interessant ist das verhältnismäßig starke Vorkommen der Höhlenbären zuletzt im Pleistozän. Ihre große Menge ist also nicht nur auf die Sedimente des R/W und W 1/2 beschränkt, sie zeigen sich fast in der gleichen Anzahl auch in 
der Schicht des W2. Es ist dies jedoch auch schon der letzte Zeitabschnitt ihres großen Vorkommens. Das gleiche gilt auch von der Höhlenhyäne und dem Wollhaarigen Nashorn.

Die Fauna des zweiten Würm-Interstadials ist ähnlich jener des vorangegangenen Zeitabschnittes. In diesem wurden folgende Tiere aufgefunden: Crocuta spelaea, Ursus spelaeus, Ursus arctos, Tichorbinus antiquitatis, Rangifer tarandus, Equus germanicus. Diese Aufzählung der Arten ergibt jedoch keine Vorstellung über deren quantitative Anzahl, die sich vor allem bei dem Höhlenbären, dem Wollhaarigen Nashorn und der Höhlenhyäne verringert.

Eine ähnliche Situation zeigt sich im W 3. Nur die Zahl der Rentiere vergrößert sich enorm. Sowohl der Höhlenbär als auch das Wollhaarige Nashorn verharren bis in diesen Zeitabschnitt.

Aus dieser knappen Aufzählung ersehen wir, daß sich nicht nur die morphologischen und metrischen Eigenschaften einiger Arten verändern, sondern daß sich während dieses Zeitabschnittes die ganze Faunen-Gemeinschaft wandelt. Bei entsprechender Menge der Fauna ist es möglich, sie zu einer detaillierten Stratigraphie der einzelnen Würm-Horizonte auszuwerten. Typisch ist die Anderung der Faunen-Gemeinschaft nach der Bildung der letzten Schwarzerde.

Für wichtig halte ich es, die Fauna aus dem W $1 / 2$ anzuführen, zumindest jedoch jene von den bedeutendsten Fundstätten. Dabei beschränke ich mich auf jene zunächst gelegenen wichtigen Lokalitäten, die im benachbarten Osterreich und Ungarn liegen. Aus der festgestellten Faunen-Gemeinschaft zeigt sich, wie nahe sich die Sedimente des R/W und W 1/2 hinsichtlich der Fauna stehen.

Eine der bedeutendsten Fundstätten in Ungarn ist die I stállóskö-Höhle. Sowohl die untere Kulturschicht (Aurignacien I) als auch die obere Kulturschicht (Aurignacien II) und weiter die gelbbraune lößartige Schicht in ihrem Hangenden ist dem Interstadial W $1 / 2$ einverleibt. Die wichtigsten Tiere, welche in der unteren Kulturschicht gefunden wurden, sind: Canis lupus, Vulpes vulpes, Ursus spelaeus, Ursus arctos, Meles meles, Martes martes, Hyaena spelaea, Panthera spelaea, Lynx lynx, Mammonteus primigenius, Cervus elaphus, Rangifer tarandus, Capra ibex, Bison priscus, Rupicapra rupicapra, Lepus timidus u. ä. Die Fauna der oberen Kulturschicht ist sehr ähnlich. Es waren alle oben angeführten Arten vorhanden; von weiteren kam beispielsweise der Elch hinzu. Das gleiche können wir von der gelbbraunen lößartigen Schicht sagen, in der aus der Reihe der oben angeführten Tiere der Dachs, die Höhlenhyäne und der Elch fehlen, hingegen aber das Wildschwein und die Wildkatze vorkommen.

Dieses Material ist in den einzelnen Arten dem des Švédův stůl sehr ähnlich, entspricht aber schon nicht mehr dem seiner Menge.

Eine weitere wichtige Lokalität ist Willendorf in Ósterreich. Dort finden sich sowohl die große Form des dem Maral ähnlichen Hirsches, als auch Bison und Elch. Leider ist bei der Lokalität Wille ndorf I das Material nicht präzis stratifiziert, und aus den übrigen Aufschlüssen dieser Lokalität gibt es relativ wenig. Interessant ist, daß in der Lokalität Willendorf I vereinzelt nicht stratifizierte Reste der Art Dicerorbinus kirchbergensis auftreten.

Von weiteren Fundstätten, deren paläontologisches Material ich aber nicht mehr anführen werde - das aber dem der vorangeführten Lokalitäten vollständig ähnlich ist sind es in Ungarn die Höhlen Szeleta, Lökvölgyer, Kiskevélyer, Balla, Mexicovölgyer, Hermann-Höhle, Csákvárer, Szelim, Diosgyör u. a. In Deutschland ist dies noch z. B. W a $11 \mathrm{e} \mathrm{rth}$ e i m.

Alle bereits angeführten Faunen-Gemeinschaften und deren im Verlauf des Letzten Glazials eingetretenen Veränderungen zeigen, daß nach dem W 1/2 eine große klimatische Anderung eintrat. 
Eine ähnliche Faunen-Gemeinschaft finden wir auch in der $\mathrm{K} \mathrm{r}$ a p in a, wo vor einigen Jahren die Sedimente in das erste Würm-Interstadial gestellt worden sind, wobei die Fauna vollständig dem Interglazial, z. B. der Fauna von T a ub a h, gleicht. E. W. Guenther schreibt richtig in seiner Arbeit (1959), daß diese Gemeinschaft in beiden Zeitabschnitten hätte leben können, d. h. sowohl im R/W wie auch im W 1/2. Die vorgefundene Fauna setzt sich aus folgenden Arten zusammen: Ursus spelaeus, Dicerorhinus kirchbergensis, Castor fiber (am häufigsten). Weniger häufig sind Bos oder Bison, Ursus arctos, Canis lupus, Cervus elaphus, Marmota marmota vertreten. Zumindest durch ein Tier sind nachstehende Arten erwiesen: Capreolus capreolus, Sus scrofa, Equus sp., Megaloceros giganteus, Felis silvestris, Myoxus glis, Mustela foina, Lutra lutra, Cricetus frumentarius, Elephas sp. und Mus sp. In größter Menge wurden die Nashörner gefunden.

Zwischen der angeführten Fauna aus $\mathrm{Kr}$ a p in a, die zeitlich W 1/2 darstellen soll, und der Fauna aus dem interglazialen Travertin aus der Weimarer Umgebung, gegebenenfalls auch der Fauna des Švéd ův st ůl, besteht in den Arten praktisch kein Unterschied. Soweit es Dicerorbinus kirchbergensis im W $1 / 2$ anlangt, bildet $\mathrm{K}$ r a p in a allerdings nicht irgendeine Ausnahme. Aus Sedimenten der gleichen Altersstufe wurde dieser von Rakovec aus der Höhle $\check{C} \mathrm{rni} \mathrm{kal}$ angeführt, weiter aus den Funden in der $\mathrm{R}$ o m a n e $11 \mathrm{i}-$ Höhle in Italien nachgewiesen usw. Allgemein nimmt man heute an, daß in den Gegenden südlich der Alpen diese Art nicht nur im Letzten Interglazial, sondern auch noch im ersten Würm-Interstadial vorkommt. Im nördlichen Spanien wurden solche Funde in den Sedimenten aus der Höhle bei der Stadt Santander (C u e v e del C a s til lo) sogar noch aus dem W 2 angeführt; aus dem Aurignacien ist sie auch aus Fundstätten in der Umgebung der Pyrenäen angegeben.

Das bedeutet, daß die paläontologische Unterscheidung der beiden Schichten (R/W und W 1/2) mindestens in den angeführten Gegenden sehr schwierig, manchmal sogar auch unmöglich ist, weil die Faunen-Gemeinschaft der beiden Zeitabschnitte fast identisch ist.

Die Anzahl der Diskussionsbeiträge zur Stratigraphie des letzten Glazials ist seit dem Erscheinen des Artikels von H. L. Movius in "Current Anthropology“ (1960) stark angestiegen. Trotz der Verschiedenheit der Ansichten scheint es mir möglich, eine Grundlage zu finden, auf der sich die Meinungen miteinander in Einklang bringen ließen. Die Mehrzahl der Autoren stimmt überein in bezug auf die chronologische Einreihung des letzten Würm-Stadials W 3, des Interstadials W 2/3 (Paudorf) und der Lößablagerung W 2. Ich glaube, daß die Ansichten von J. KukLA \& B. KLímA (1961) hinsichtlich der Dauer des letzten Interstadials nicht richtig sind. Auch H. Gross (1961) und H. L. Movius (1961) widerlegen sie in überzeugender Weise.

$\mathrm{Zu}$ diametral voneinander abweichenden Ergebnissen führten jedoch die Studien über den Zeitabschnitt W 1/2 (Göttweig). Die Meinungsverschiedenheiten betreffen vor allem die Eingliederung dieses Abschnitts in das Pleistozänschema, die Dauer und den Charakter des Klimas. Ohne auf die verschiedenen Ansichten einzugehen, will ich mich gleich mit den Ergebnissen befassen, die die Forschungsarbeiten auf dem Gebiet der Tschechoslowakei erbracht haben. Wir können auf einige unwiderlegliche Feststellungen verweisen. In den Lößprofilen ist der Boden gewöhnlich von zwei Lößschichten überdeckt, die durch eine Verlehmungszone W 2/3 voneinander getrennt sind. Infolge Solifluktion bzw. Abschwemmung pflegt die Oberfläche feinschichtig zu sein. Unter dem Boden finden wir eine starke Lößschicht, die in einigen Fällen durch schwache Humusschichten getrennt ist. Diese Entwicklung stellen wir nicht nur in freiliegenden Abräumen fest, sondern auch in Höhlensedimenten. Das Alter einiger Böden dieses Zeitabschnittes wurde auch mit Hilfe von $\mathrm{C}^{14}$ bestimmt. Es handelt sich um die Böden in Dolní Věstonice, die über 52000 Jahre alt sind (Kukla \& K Kíma 1961), und in M a lo m ěr i c e, wo sie im Hangenden einer (10 $\mathrm{m}$ hoch liegenden) Terrasse liegen und von den betreffenden Lössen überdeckt sind (MusIL \& V ALOCH 
1961). Das Alter der Böden von Maloměřice liegt ebenfalls bei über 53000 Jahren. Von den Höhlensedimenten sind es die Böden der Höhle Pod hrade m, deren oberer Teil $32000 \pm 1500$ Jahre alt ist. V. LožEK findet in diesen Böden die Reste von Steppenschnekken (in Gross 1961, p. 359). Allerdings fand er später bei der Erforschung von D oln í V ěs to n i c e auch die Reste thermophiler Fauna (in KukLA \& KLíma 1961, p. 439). Es bestehen also offensichtlich Lokalitäten, deren Boden Reste von Steppenfauna enthält, und Lokalitäten mit Resten typisch interglazialer thermophiler Fauna. Es ist klar, daß diese Feststellungen die oben angeführten Autoren dazu veranlaßt, den unteren Teil (LessivéBöden) dem R/W anzureihen, den oberen 'Teil dann der Göttweiger Verlehmung (in Gross 1961) oder den ganzen Boden in das Letzte Interglazial einzugliedern.

Diese Ansicht stützt sich auch auf die Erforschung des Š véd ův st ůl (Schwedentischgrotte), dessen Boden zwar nicht mit Hilfe von $\mathrm{C}^{14}$ datiert werden konnte, doch in paläontologischer Hinsicht das gleiche Bild vermittelt. Im unteren Teil ist der Bodenkomplex interglazialen Ursprungs, der obere Teil gehört dem W $1 / 2$ an. Úber diesem Bodenkomplex liegt dann, charakteristisch entwickelt, der übrige Teil des Würms.

Auf Grund der bisherigen paläontologischen Auswertung und der absoluten Chronologie nehme ich an, daß alle Böden dieses Zeitabschnitts, soweit sie in situ vorgefunden werden, ein Alter von über 50000 Jahren aufweisen werden. Bei Böden von geringerem Alter - interessanterweise sind es hauptsächlich Höhlensedimente - handelt es sich um verlagerte Böden, wo Bildung und Verlagerung zeitlich nicht zusammenfallen (Pod hradem $32000 \pm 1500$, Salzofenhöhle $34000 \pm 3000$, Istallóskö $30170 \pm 600$ ). Ich teile hier voll die Ansichten von K. J. NARR (1961), der diesen Zeitabschnitt als „Early Glacial Period" bezeichnet, und von F. J. Brandtner (1961). Es wäre nicht richtig, diesen angeschwemmten Böden dieselbe Bezeichnung zu geben wie den Böden in situ. Wenn auch das Alter der Böden das gleiche ist, liegt zwischen der Entstehung und der vor den Lößanwehungen des W 2 erfolgten Verlagerung doch ein bedeutender Zeitraum.

Die wichtigste Frage ist allerdings die Altersbestimmung des unteren Bodens und damit auch des liegenden Lösses, der von uns als $W_{1}$ (Warthe) bezeichnet wurde. Es ist dies eigentlich die letzte pleistozäne Bildung von Schwarzerde, die wir laufend in allen Lößprofilen vorfinden; das Liegende enthält meist eine mächtige Lößschicht, unter der ein ganzer Komplex von Böden liegt.

Die Erforschung des Švéd ̊̊v st ů 1 scheint die Ansicht zu stützen, daß es sich um das Letzte Interglazial handelt. Trotzdem glaube ich, daß die ganze Problematik dieses Abschnitts noch einer weiteren und gründlicheren Klärung bedarf, bevor dieser Annahme definitive und allgemeine Gültigkeit zugesprochen werden kann. Erinnern wir uns nur z. B. des begrabenen Bodens von Ehringsdorf, der im Hangenden von interglazialen Travertinen liegt und der sich höchstwahrscheinlich zur selben Zeit gebildet hat wie die letzte pleistozäne Schwarzerde bei uns. Denken wir auch daran, daß in den Sedimenten, die wir als Bodenkomplex aus dem R/W bezeichnen, Molarzähne des $M$. primigenius aufgefunden werden. Ich nehme an, daß zur chronologischen Einreihung dieses Abschnitts noch die Erforschung weiterer Profile erforderlich sein wird, vor allem solcher, wo beide Schichten, d. h. die heutigen $W 1 / 2$ und $R / W$, durch Löß getrennt, übereinander liegen und paläontologisch gut fundiert sind.

Aus diesen sehr kurz angeführten Schlußfolgerungen ersehen wir, welche Probleme sich bei der Frage der Festlegung des Zeitpunktes der Schichten aus dem Letzten Glazial ergeben. In der Mehrzahl handelt es sich um Probleme grundsätzlichen Charakters. Es wird notwendig sein, die Aufmerksamkeit offenkundlich auf die Faunen-Gemeinschaften des Letzten Interglazials und des ersten Würm-Interstadials zu lenken, da diese Zeitabschnitte heute den Schwerpunkt der wichtigsten Probleme des Jungpleistozäns bilden und weil hier offensichtlich viel mehr Unklarheiten vorhanden sind, als nach dem ersten Anschein zu erwarten waren. 


\section{Literatur-Verzeichnis}

Brandtner, F. J., 1961: Comments to H. L. Movius 1960. - Current Anthropology 2, p. 427-428. EHRENBERG, K., 1928: Betrachtungen über den Wert variationsstatistischer Untersuchungen in der Paläozoologie nebst einigen Bemerkungen über eiszeitliche Bären. - Paläont. Zeitschrift 10, p. 235-257, Berlin.

Gross, H., 1961: Comments to H. L. Movius 1960. - Ibidem, p. 428-434.

GuENTHER, E. W., 1959: Zur Altersdatierung der diluvialen Fundstelle von Krapina in Kroatien. Bericht über die 6. Tagung d. Deutsch. Ges. f. Anthropologie, p. 202-209.

Kukla, J. \& KLíma, B., 1961: Comments to H. L. Movius 1960. - Ibidem, p. 437-439.

Movius, H. L., 1960: Radiocarbon Dates and Upper Palaeolithic Archaeology in Central and

Western Europe. - Current Antrop. 1, p. 355-375.

Movius, H. L., 1961: Reply. - Ibidem 2, p. 428-434.

Musil, R., 1961: Die Höhle „Švédův stůl“ - ein typischer Höhlenhyänenhorst. - Anthropos 13 (N.S. 5), p. 97-260, Brno.

MusiL, R., 1961: Die unteren Terrassen der Svitava bei Brünn. - Práce Brněnské základny Čs. Akad. věd. 33, p. 225-256, Brno.

NarR, K. J., 1961: Comments to H. L. Movius 1960. - Current Anthrop. 1, p. 444-448.

SoERgel, W., 1937: Die Stellung der Hyaena spelaea Goldf. aus der Lindentaler Hyänenhöhle bei Gera. - Beiträge Geol. Thüringen, Bd. 4, H. 5, p. 171-189.

SoERGEL, W., 1930: Die Bedeutung variationsstatistischer Untersuchungen für die Säugetier-Paläontologie. - N.Jb. f. Min. etc., Beil. Bd. 43, Abt. B, p. 349-450, Stuttgart.

Thenıus, E., 1959: Die jungpleistozäne Wirbeltierfauna von Willendorf i. d. Wachau, N.Ö. Mitt. d. Präh. Komm. 8-9, p. 133-167, Wien.

Rode, K., 1930: Über den ersten Unterkiefermolaren bei Bären. - Zentralbl. f. Min. etc., Abt. B, p. 83-90, Stuttgart.

Manuskr. eingeg. 14. 2. 1961.

Anschrift des Verf.: Dr. Rudolf Musil, Moravské museum Brno, Tschechoslowakei. 